\title{
Información Geográfica Voluntaria (IGV), estado del arte en Latinoamérica
}

\author{
Aldo I. Hernández Magaña* \\ Frida N. Güiza Valverde**
}

Recibido el 05 de abril de 2016; aceptado el 25 de septiembre de 2016

\begin{abstract}
The aim of this paper is to review the state of the art of Volunteered Geographical Information in Latin America. This is an emerging area of Neogeography, it studies the way citizens obtain and voluntarily provide information on specific problems of their interests to other social agents, using technologies and the web. The most relevant aspect of VGI is the integration of information technologies of communication with GPS, allowing the creation of cartographies based on particular interests of the users. This literature review presents the key areas of interests for LatinAmerican scientist regarding the field of VGI. Topics such as the epistemological perspective of VGI and the Neogeography; spatial data infrastructure; data quality and validation; participative cartography and disaster risk reduction are discussed in this paper.
\end{abstract}

Key words: Volunteered Geographical Information, Latin America, ITC-G's, citizen participation.

\section{Resumo}

O objetivo deste artigo é fazer uma revisão sobre o campo de Informação Geográfica Voluntária na América Latina. Esta área de estudo surgiu recentemente (início de 1980), está no campo de estudo da Neogeografia e visa estudar a troca de informa-

* Licenciado en arquitectura, maestría en Manejo Integrado del Paisaje, Centro de Investigaciones en Geografía Ambiental (CIGA), Universidad Autónoma de México (UNAM), correos electrónicos: ahernandez@pmip.unam.mx, arq.aldo.hernandez@gmail.com

** Investigadora asociada, Centro de Investigaciones en Geografía Ambiental, Universidad Autónoma de México, CONACYT/CIGA-UNAM, correos electrónicos: frida.ciga@gmail.com, fguiza@ciga.unam.mx Los autores agradecen los comentarios de los revisores de este artículo, los cuales contribuyeron a mejorar significativamente este trabajo. 
ções, o uso, a participação e apropriação da informação, tais como uso de dispositivos tecnológicos por parte dos cidadãos e outros atores sociais atuantes. $\mathrm{O}$ aspecto mais importante deste campo é a integração do desenvolvimento tecnológico no domínio da Internet, das Tecnologias de Informação e Comunicação, onde se integra a componente espacial através de GPS, permitindo a geração de mapas que representam os interesses individuais dos usuários. Esta revisão da literatura apresenta os interesses mais relevantes da academia na América Latina sobre o tema da informação geográfica como: análise epistemológica da neogeografia; infraestrutura de dados espaciais (SDI), qualidade e validação de dados; a cartografia participativa e, particularmente a gestão de desastres e risco, áreas que se desenvolveram na América Latina.

Palavras-chave: Informação Geográfica Voluntária (IGV), América Latina, TIC-G, Participação, Cidadãos.

\section{Resumen}

El objetivo de este artículo es hacer una revisión de la literatura que sobre el campo de la Información Geográfica Voluntaria hay en Latinoamérica. Esta área de estudio de reciente emergencia (década de los ochenta), se encuentra en el ámbito de estudio de la Neogeografía y se orienta a estudiar el intercambio, uso, participación y apropiación de la información, como de los dispositivos tecnológicos por parte de los ciudadanos y otros agentes sociales interactuantes. El aspecto más relevante de este campo es la integración del desarrollo tecnológico en el ámbito de la Internet, las Tecnologías de la Información y Comunicación, a las que se agrega el componente espacial del GPS, lo cual permite la generación de cartografías que representan los intereses particulares de los usuarios. Esta revisión de la literatura presenta los intereses más relevantes de la academia en Latinoamérica sobre el tema de la información geográfica como: y el análisis epistemológico de la neogeografía; la infraestructura de datos espaciales (IDE), la calidad y validación de datos; la cartografía participativa y particularmente la gestión de desastres y riesgos son áreas que, se desarrollan en América Latina.

Palabras clave: Información Geográfica Voluntaria (IGV), Latinoamérica, TIC-G's, participación, ciudadanos.

\section{Introducción}

El uso generalizado de nuevas tecnologías de la información y comunicación geográfica (TIC-G), ${ }^{1}$ representa un cambio en la perspectiva de la generación y georre-

TIC-G, acrónimo que desde nuestra perspectiva responde de manera más precisa a las particularidades de la IGV, dado que vincula los componentes tecnológicos de a comunicación y la espacialización. 
ferenciación de datos porque implica a los usuarios. Las TIC-G integran avances técnicos como la geovisualización articulada con la Internet en tecnologías de bolsillo, así como la generación de información, cuya característica más relevante es la movilidad, fácil portabilidad y la generación de información desde los ciudadanos.

En el caso latinoamericano algunos autores como Buzai (2014), Mateos (2013) y Orellana y Ballari (2009) hacen uso de términos como: nuevas geografías, neogeografía, geografía de las masas, ambientes participativos y colaborativos, personas como sensores o información geográfica voluntaria, para contextualizar un cambio de paradigma de la participación ciudadana, posibilitada por el componente tecnológico. Las capacidades tecnológicas de estos instrumentos permiten a los ciudadanos exponer problemáticas locales con el apoyo de servicios de producción de información georreferenciada (Mateos, 2013).

La naturaleza del desarrollo tecnológico y la integración amigable de características como: servicios basados en la web 2.0, dispositivos equipados con sistemas de posicionamiento global (GPS) y el desarrollo de la geovisualización en dispositivos móviles, permite la apropiación de parte de los usuarios en la generación, provisión y difusión de información. El carácter interactivo de las tecnologías provee una versión ciudadana de la realidad, que se asume puede complementar las perspectivas del mundo académico y los profesionales. A esta transformación del paradigma de la participación ciudadana basada en la tecnología actual, se ha llamado democratización de la información geográfica (Buzai, 2014; Mateos, 2013; McCall, 2011).

En este artículo se hace una revisión de la literatura latinoamericana con el objetivo de entender los diferentes componentes e influencias de lo que llamamos: el marco analítico de la Información Geográfica Voluntaria, IGV o VGI (Volunteered Geographic Information por sus siglas en inglés), la intención es conocer los avances en la región con respecto a un tema que se desarrolla vertiginosamente en el mundo y del que no está desligado Latinoamérica.

Este articulo está organizado en cuatro grandes apartados: el primero, compila los diferentes componentes e influencias teóricas y conceptuales de la IGV desde la perspectiva latinoamericana; en el segundo, se explican las estrategias de recolección y análisis de la literatura sobre el tema, así como los elementos que se consideraron para la delimitación tanto temporal como temática de la literatura disponible en Latinoamérica; en el apartado tercero, se hizo la discusión general resultado de la revisión de la literatura en la cual se plantearon y discutieron los enfoques, teorías y conceptos relacionados con la IGV predominantes en Latinoamérica; finalmente en el cuarto apartado se desarrollan conclusiones sobre el uso de la IGV y los retos que implica su uso. 


\section{Marco teórico y conceptual de la Información Geográfica Voluntaria}

El concepto de la IGV hace referencia a la provisión de información geográfica a partir de la participación voluntaria de ciudadanos. Los cuales se convierten en sensores que recogen información de manera selectiva y dirigida (inteligente), para la generación de conocimiento. Este modelo provee voluntariamente de conocimiento local, o en términos de Cook (2005) situado. Esta información provista desde los ciudadanos y de manera situada es relevante para el diseño y toma de decisiones. El acceso creciente a dispositivos móviles como celulares, tabletas o computadoras portátiles implica que esta información sea generada en tiempo real. Ejemplos de estos sistemas colaborativos pueden encontrarse en plataformas como Wikimapia, Open Street Map, Google earth, Google maps, Ushahidi y actualmente en redes sociales de carácter comercial como Facebook y Twitter, que permiten crear, proveer y difundir información geográfica de forma voluntaria (Goodchild, 2007b; Goodchild, 2007a). En relación al tema de la provisión consensuada y deliberada de información existen una serie de cuestionamientos que serán abordados más adelante.

La Información Geográfica Voluntaria (IGV) se compone de tres elementos principales: primero, las personas que de manera deliberada recolectan y suministran información, lo cual los convierte en sensores. Un segundo componente son las tecnologías de la información y la comunicación geográfica (TIC-G), Las TIC-G permiten una interacción amigable del usuario como generador de información con servicios de geovisualización y de difusión. Un tercer componente es la generación de datos e información geográfica. Este último componente es fundamental y es el que asigna nombre a esta forma de participación voluntaria en la recolección y suministro de información. Estos tres elementos de acuerdo con Goodchild (2007b; 2007) y Mateos (2013) dan sentido a la IGV.

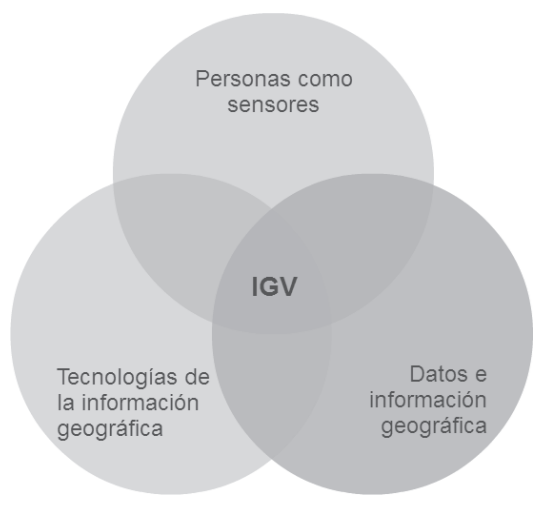

Figura 1. Elementos que componen la información geográfica voluntaria IGV. Elaboración propia. 
La convergencia de conocimientos en los ámbitos social, tecnológico y de sistemas computacionales se genera por la rapidez en el desarrollo, uso y apropiación social de la tecnología, la comprensión a profundidad de los elementos arriba mencionados (véase Figura 1) es fundamental para entender la interface IGV, y generar un análisis más sólido que facilite su uso y consolidación dentro de la corriente teórica de la neogeografía, como de las otras disciplinas.

Tabla 1

Contribuciones a la IGV de diferentes corrientes teóricas

\begin{tabular}{ll}
\hline \multicolumn{1}{c}{ Propuestas teóricas } & \multicolumn{1}{c}{ Contribuciones en la IGV } \\
\hline Neogeografía & $\begin{array}{l}\text { Análisis crítico del uso y apropiación de tecnologías de la } \\
\text { información y comunicación geográfica }\end{array}$ \\
Ciudadanos como sensores & $\begin{array}{l}\text { Fundamentos epistémicos a la IGV } \\
\text { Inclusión y democratización del conocimiento generado } \\
\text { Cartografía participativa }\end{array}$ \\
$\begin{array}{l}\text { Tecnologías de la información y local y participativa } \\
\text { comunicación geografía (TIC-G) } \\
\text { Información geográfica }\end{array}$ & $\begin{array}{l}\text { Calidad y validación de los datos } \\
\text { Casos de estudio, gestión de desastres y riesgos }\end{array}$ \\
\hline
\end{tabular}

Fuente: Elaboración propia.

La Tabla 1 presenta las contribuciones teóricas al cuerpo conceptual y analítico de la IGV que se encontró predominan en esta revisión de la literatura latinoamericana. Desde nuestra perspectiva, reconocer el estado actual del conocimiento generado en relación a la $\mathrm{IGV}$ en la región es relevante, dado el creciente uso, generación de información y las mejoras técnicas de los dispositivos móviles. Desde la academia saber los aspectos relevantes para los académicos, nos permite reconocer los límites del conocimiento generado hasta el momento, y avanzar en la generación de otros nuevos al detectar vacíos en la investigación. Esta revisión de la literatura, pretende mostrar los alcances actuales de la IGV en Latinoamérica, por lo que se plantea responder a las siguientes preguntas: ¿Cuáles son los avances en términos teóricos y prácticos reportados en la literatura?, ¿cómo se desarrolla la IGV?, ¿cuáles son los enfoques de análisis teóricos en relación a la IGV que predominan?, ¿es relevante la propuesta de la IGV para la disciplina geográfica y cartográfica?, ¿la IGV como estrategia de colección de información y como marco analítico es funcional?

En esta revisión se observa la producción de literatura desde las distintas posturas y enfoques de los autores, así como la evolución e interacción en la conforma- 
ción del marco analítico de la IGV. Si bien la presencia de estudios sobre la IGV tiene menos de una década, la producción de conocimiento en Latinoamérica se puede considerar como emergente, cobrando cada vez mayor interés para actores privados, instituciones gubernamentales y la academia.

\section{Métodos}

La revisión de la literatura sobre IGV, se construyó con siguiendo una estructura temporal y temática, que permitió tener un panorama amplio de la información generada en el ámbito académico referente a la IGV en Latinoamérica. Para la búsqueda de la literatura se determinó un horizonte temporal, fijando como inicio el año 2007, y como límite al mes de febrero del año 2016. Para este caso sólo se tomaron en cuenta aquellos documentos que fueron publicados dentro de esta temporalidad.

Adicionalmente se estableció que sólo serían parte de esta revisión aquellos documentos y literatura que fuera realizada en el ámbito académicos por estudiantes, investigadores y/o grupos de investigación adscritos a algún centro de investigación, universidad o instituto que se localizase en Latinoamérica, esto con la intención de tener un rango acotado de la producción del conocimiento académico.

La búsqueda de literatura se realizó por medio de la frase o palabra clave "información geográfica voluntaria”, así como su abreviación por sus siglas (IGV). Se realizaron búsquedas de documentos en inglés y portugués, los cuales son los idiomas predominantes para la publicación de documentos en Latinoamérica, la ubicación de la palabra clave podría encontrarse en el título, en palabras clave y/o en el contenido del documento.

La localización de documentos estuvo basada en la web. Se utilizaron buscadores como: Google search, Google académico, bases de datos de revistas científicas latinoamericanas como Latindex, Scielo, Redalyc, bases de datos internacionales como SCOPUS, y la base de datos proporcionada por la biblioteca de la Universidad Nacional Autónoma de México.

Una vez realizada la búsqueda de documentos se procedió a analizar los temas tratados en los documentos. Se generó una base de datos progresiva, que permitiera discriminar la literatura que no cumpliera con los criterios adoptados en esta revisión de literatura. La base de datos se construyó con la siguiente información: autores, título, palabras clave, afiliación académica, año de publicación, tipo de documento, buscador utilizado.

Como resultado de la búsqueda en las diversas bases de datos antes mencionadas, se encontró un total de 48 documentos procedentes de revistas científicas, conferencias, libros, reportes y tesis en América Latina sobre el tema. De estos documentos encontrados, se procedió a la discriminación de la información, por lo 
que se eliminó aquella literatura que no se centraba en la IGV, es decir literatura que no presentara análisis y/o uso de la IGV. Como resultado obtuvimos la Tabla 2 , que se presenta a continuación.

Tabla 2

Documentos encontrados por objeto de estudio

\begin{tabular}{lcr}
\hline \multicolumn{1}{c}{ Tema } & $\begin{array}{c}\text { Periodo } \\
\text { de estudio }\end{array}$ & $\begin{array}{c}\text { Cantidad de } \\
\text { documentos }\end{array}$ \\
\hline Neogeografía & $2009-2013$ & 4 \\
Infraestructuras de datos espaciales & $2012-2015$ & 7 \\
Casos de estudio, gestión de desastres y riesgos & $2012-2015$ & 13 \\
Cartografía participativa & $2013-2015$ & 6 \\
Información geográfica voluntaria (epistemología) & $2015-2015$ & 1 \\
Calidad y validación de los datos & $2015-2016$ & 8 \\
\hline \multicolumn{1}{c}{ Total } & 39 \\
\hline
\end{tabular}

Fuente: Elaboración propia.

La Tabla 2 presenta el resultado de la búsqueda de literatura referente a la IGV, el orden de la tabla responde al horizonte temporal fijado para los artículos seleccionados. En la tabla se observa le creciente interés sobre el tema, a partir de la producción de literatura en el año 2009. Desde nuestra perspectiva este desarrollo cronológico muestra la congruencia en el desarrollo de la temática de la IGV en la región, basado en el estudio de la neogeografía y el acceso a las tecnologías móviles.

La condición anterior estimuló el interés en el conocimiento de las IDE, para facilitar la interacción entre las plataformas y los usuarios, particularmente en casos de desastres.

Un componente importante es la integración del campo de la cartografía participativa y la IGV desde la epistemología, aunque en este caso las contribuciones han sido mínimas. Por último, se observa en los últimos años un creciente interés en el tema de la validación y calidad de los datos entre los académicos latinoamericanos, aspectos que se discutirán en la siguiente sección.

\section{Hallazgos de la revisión de literatura abordajes conceptuales y corrientes teóricas}

En este apartado se presentan las principales abordajes conceptuales y corrientes teóricas que influencian la investigación de la IGV en Latinoamérica, entre las cuales destacan: la neogeografía, el uso de nuevas tecnologías de comunicación, la participación de los ciudadanos y las posibilidades de generación de datos georreferenciados. 


\section{Neogeografía}

El paradigma teórico de la neogeografía propone reconocer el carácter difuso de las fronteras entre conocimiento experto y no experto para la creación de información geográfica.

La corriente de la Neogeografía se fortalece desde fines de los años 1990 con la accesibilidad a nuevas tecnologías de la comunicación que permiten la participación activa de los ciudadanos en la generación de información. Esta condición se genera en sincronía con la falta de capacidad e infraestructura del sector gubernamental y especialistas en cartografía para generar de manera eficiente y actualizada información para el diseño y la toma de decisiones. Los principales análisis se encuentran situados en revisiones de literatura que presentan el estado del conocimiento sobre la neogeografía (Orellana y Ballari, 2009; McCall, 2011; Mateos, 2013).

Aún dentro del campo de la neogeografía McCall et al. (2015) sugieren la construcción de la IGV con una perspectiva basada en las influencias del mapeo participativo por lo cual abogan por la valorización del conocimiento local, desde esta perspectiva los ciudadanos colaboran en la generación de información geográfica. La cual comprende a los sistemas de información geográfica participativa (SIGP) y otros sistemas de contenido espacial generado por usuarios (McCall, 2011; McCall et al., 2015).

En el caso mexicano Güiza y McCall (2016); McCall (2011; 2015) centran sus estudios en analizar la IGV desde un enfoque de la geografía humana, la cual revalora el conocimiento local. En general la investigación está orientada a la generación de cartografía funcional, pero no es replicada por otros casos de estudio, dado que aun presenta retos, tema que se abordará más adelante.

\section{Ciudadanos como sensores}

En consonancia con la propuesta de la neogeografía surge la propuesta de los ciudadanos como sensores. Desde esta perspectiva los ciudadanos son partícipes en la generación de la información, apoyados de tecnologías que permiten su recopilación. Ejemplos de esto se pueden encontrar en aplicaciones como "VGI Morelia" la cual fue desarrollada en México con el fin de que los usuarios reportaran problemáticas ambientales y sociales a las autoridades, a través de celulares. Esta aplicación complementa el envío de información con un componente espacial cartográfico y georreferenciado que está disponible para la población que tiene acceso a estas tecnologías (Güiza y McCall, 2016).

En un análisis más detallado sobre el funcionamiento de los ciudadanos como sensores, Stasch et al. (2009) sugieren que hay diferencias significativas entre el uso de sensores técnicos para estudiar fenómenos complejos de la naturaleza por su debilidad para moverse fluidamente a diferentes escalas, lo cual implica perdida de 
información de tipo espacial como de tipo sensorial y perceptivo que naturalmente generan los seres humanos, información que puede ser integrada a partir de la aplicación del uso de tecnologías aplicadas a la IGV. La relevancia de estos estudios se encuentra en el análisis de las capacidades de los ciudadanos sensores, los cuales recopilan de manera integral una diversidad de información como percepción de temperatura y color, densidad de un elemento y ruido, a diferentes escalas, lo que posiciona al ciudadano como sensor en un potencial proveedor de información.

Particularmente en Brasil, autores como Hirata et al., 2013; Vidal-Filho et al., 2013; Atzmanstorfer et al., 2014; Eckle y De Albuquerque, 2015; Bravo et al., 2015, han encontrado en la IGV un potencial para la generación de información geográfica. Estos investigadores desarrollan marcos conceptuales para el mapeo colaborativo, usando servicios de geovisualización, para la producción de cartografía basada en la IGV. Así mismo, hacen uso extensivo de las redes sociales como proveedores de datos para la actualización de bases de datos cartográficos oficiales.

Es importante señalar que en México como en otros países, hasta hace muy poco la legislación sobre el uso y privacidad en el manejo de datos, era ambiguo. En los estudios antes citados esta condición aparece imprecisa en la parte metodológica. En suma, los principales aportes en el ámbito de los ciudadanos como sensores y la IGV en América Latina se han desarrollado en Brasil, particularmente en el ámbito tecnológico, con un enfoque en los sistemas computacionales, aunque se observa investigación desarrollada en la esfera social en la cual se analiza el rol del ciudadano y las ventajas que aporta su inclusión en la investigación.

\section{Datos e información geográfica}

Los datos y la información geográfica son elementos claves en la IGV. De acuerdo con esta revisión de la literatura, en América Latina la IGV se ha desarrollado preponderantemente en el área de los sistemas computacionales; particularmente los metadatos dinámicos, diccionarios de datos y análisis cuantitativos como apoyos para la geocodificación de la información Por ejemplo, Davis y De Alencar (2011) proponen el uso de la IGV como recurso alternativo a métodos de recolección de datos pensados en contextos que no corresponden a la realidad de nuestras ciudades. Por ejemplo, la geocodificación de bases de datos de domicilios en ciudades latinoamericanas, basadas en la IGV resulta ventajoso, por la falta de información e infraestructura para su generación. Hasta el momento las metodologías se basan frecuentemente en modelos que no corresponden a las condiciones de ciudades latinoamericanas, particularmente se basan en el modelo estadunidense, en el que las direcciones se asocian a segmentos o cruces viales. En el caso latinoamericano es información que no está disponible en bases de datos porque la urbanización se da de manera informal y poco planeada, en estos casos el uso de la IGV mejora la fiabilidad y disponibilidad de la información. 
De especial interés en esta revisión de literatura es la validación de los datos. En este ámbito los esfuerzos se enfocan en la construcción metadatos, definidos como estructuras para la administración de datos. Los estudiosos en estos temas construyen sistemas que permiten establecer normas y estándares para facilitar la recolección, documentación y validación de datos de la IGV (Souza et al., 2013; De Souza et al., 2014).

Vidal-Filho et al. (2013), proponen el uso de diccionarios cartográficos como herramientas para validar datos provistos a partir de la aplicación de la IGV, frente a datos oficiales como censos. Por ejemplo, un problema fundamental al cruzar la información recogida por la IGV y los Censos es la estandarización semántica y estructural de datos provenientes de fuentes diferentes. Algunos elementos que sugieren los autores para validar datos provenientes de contribuyentes (ciudadanos) son: la evaluación rigurosa del dato proveniente de un usuario; la trasparencia sobre quien es el usuario; el número de contribuyentes que validan la información. Estos elementos pueden por un lado mejorar la calidad del dato provisto a través de la IGV y por otro, permitir la integración de semántica (De Oliveira et al., 2015; Cardoso et al., 2016).

En síntesis, la literatura desarrollada en América Latina sobre datos e información geográfica, se orienta a promover iniciativas relacionadas con la IGV como generadora y distribuidora de información colaborativa. Por ejemplo Linked Open Data (LOD), funciona con el modelo de anotación de datos abiertos. También se ha orientado al desarrollo de estructuras que soportan la generación de información georreferenciada, con tecnologías que generan datos legibles para aplicaciones informáticas (web semántica) (Cardoso et al., 2016).

\section{Tecnologías de la información y la comunicación geográfica (TIC-G) e Infraestructura de datos espaciales}

Los datos e información geográfica revisados arriba, se encuentran estrechamente ligados tanto a las TIC-G, como a las Infraestructuras de Datos Espaciales (IDE). Estas se definen como el conjunto de herramientas que constituyen las plataformas de visualización e interacción con los datos geográficos. El desarrollo continuo de paquetes informáticos, sistemas computacionales, servicios de geovisualización, y el acceso a tecnologías amigables con los usuarios, ha contribuido en el desarrollo y mejora de las capacidades de las IDEs, facilitando el acceso, uso, visualización, análisis y difusión de datos georreferenciados.

En la literatura latinoamericana se encontraron trabajos relacionados con el rol de las Infraestructuras de Datos Espaciales (IDE) como soporte de la IGV, para la optimización del proceso de recopilación de datos georreferenciados. Asimismo, Miranda et al., 2012; Ballari et al., (2012); Davis et al. (2013); Ballari et al. (2014) y Pacheco (2015), desarrollan y analizan software orientado a la recolección y pro- 
visión de información geográfica y cartográfica. Estos autores aplican y reconocen la IGV, sin embargo, no teorizan al respecto.

Tomando como referencia el marco de la infraestructura crítica, Herfort et al. (2015) analizan el uso de las TIC-G y las IDE para evaluar la calidad y validez de los datos recolectados y provistos a plataformas como OpenStreetMap (OSM). Estos estudios tienen una perspectiva en sistemas computacionales, y de acuerdo con esta revisión de literatura, son Brasil y Ecuador los países a la vanguardia en América Latina.

Por otro lado, en México el Laboratorio de Procesamiento Inteligente de Información Geoespacial del Instituto Politécnico Nacional realiza estudios sobre la IGV, aunque no se pudo rastrear publicaciones en plataformas académicas, si fue posible encontrar sus trabajos en la red (saber.cic.ipn.mx, 2016). Este laboratorio se orienta a la generación de plataformas que asistan en la validación y actualización de conjuntos de datos geográficos, el manejo del dominio geoespacial y la realidad aumentada.

De acuerdo con el análisis de la literatura en Latinoamérica sobre IGV, se observa el predominio de estudios orientados hacia el componente técnico y los sistemas computacionales principalmente la IDE. Resulta relevante observar que predomina el interés en el vínculo entre los sistemas computacionales y los datos generados desde la IGV y las TIC-G's. Los países en la región, donde se desarrolla mayoritariamente esta investigación son: Brasil, Ecuador y México.

Un caso de especial mención es el proyecto que desarrollan científicos mexicanos con el Instituto Nacional de Estadística y Geografía (INEGI) en México. El cual desarrolla una plataforma de validación y corrección de datos estadísticos basada en la IGV, la propuesta es que los ciudadanos contribuyan voluntariamente en este esfuerzo.

Cabe resaltar que de acuerdo a nuestra revisión de literatura, el uso datos e información obtenida desde de la IGV en Latinoamérica se ha aplicado en el campo de la gestión de desastres y riegos. Particularmente desde las disciplinas en sistemas computacionales, geoinformática y la geografía social, trabajo realizado principalmente en países como Brasil y México. Estos estudios abogan por la participación ciudadana a través de la IGV para la gestión de desastres y riesgos, en aspectos como: logística humanitaria en zonas afectas y la identificación de zonas de riesgo por inundaciones, con datos obtenidos desde la IGV. Apoyados modelos de validación de datos y sistemas que promueven un aprovechamiento eficiente de los datos obtenidos en las redes sociales (Manfre et al., 2012; Horita y De Albuquerque 2013; Horita et al., 2013; Horita et al., 2014; Herfort et al., 2014; Degrossi et al., 2014; Schelhorn et al., 2014; De Albuquerque et al., 2015; Poorazizi et al., 2015; De Brito et al., 2015; Horita et al., 2015; Peters y De Albuquerque, 2015). 
Sin duda el uso de la IGV atrae la atención de estudiosos de diferentes disciplinas, los académicos latinoamericanos desarrollan estudios enfocados a la validación de datos a través de la IGV, la generación de información geográfica basada modelos colaborativos dentro de una web semántica, la creación de metadatos y diccionarios de datos cartográficos para la estandarización de la información obtenida desde la IGV que eficientica su uso. En la parte aplicada, en la región y particularmente en Brasil y México, el desarrollo de la IGV se orienta a apoyar la gestión de desastres y riesgos. Estos trabajos se basan en las TIC-G's así como en la generación de modelos que sirvan para la validación y uso de los datos a través de los sistemas computaciones. Estos estudios aun presentan retos, y desde nuestra perspectiva demuestran que la IGV puede ser viable en el aporte de datos válidos basados en el conocimiento local, en tiempo real, una deficiencia que subsana la de otras técnicas y modelos de gestión de información.

\section{Discusión general de la revisión de literatura}

Como resultado de la revisión de literatura, se encontró que hay un interés creciente por la IGV en Latinoamérica (Tabla 3). A continuación, se discuten los resultados encontrados en la revisión de la literatura sobre IGV, esbozando las áreas de desarrollo y las áreas en las cuales existe potencial de contribuir en la generación una disciplina más sólida.

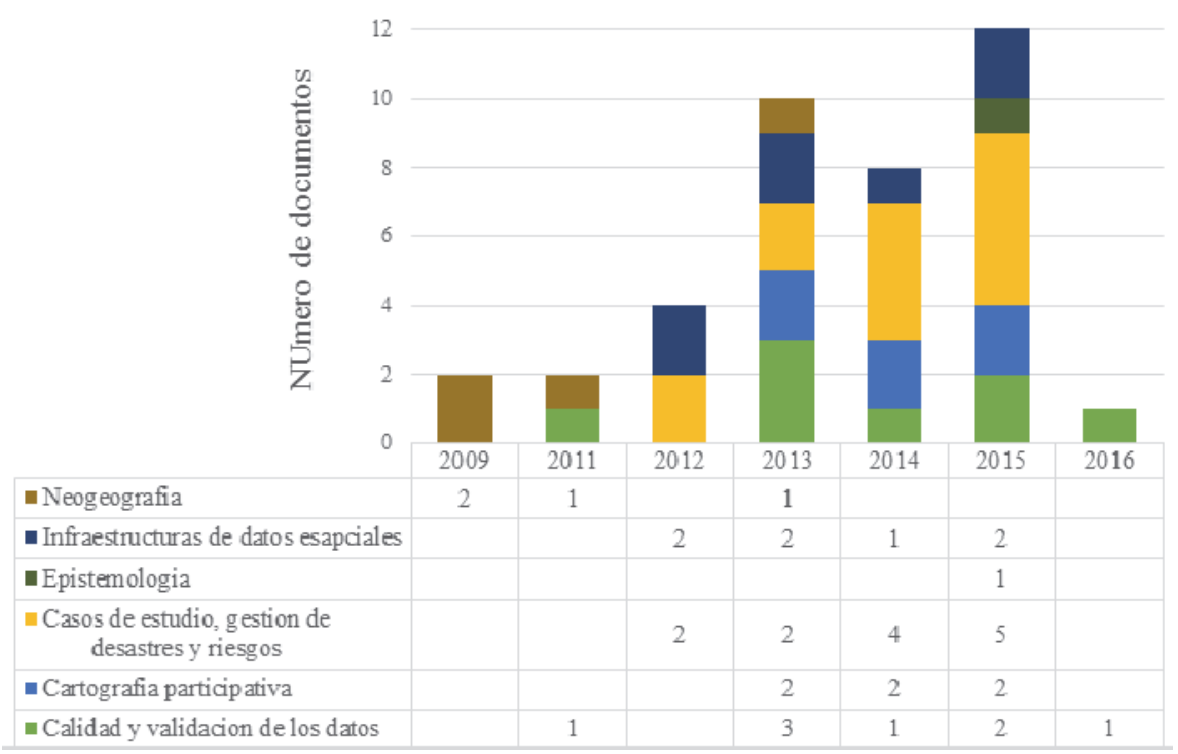

Tabla 3. Producción de literatura por año. Elaboración propia. 
A partir de los trabajos revisados, se observa que la investigación en temas de IGV en América Latina tienen un incipiente interés en la neogeografía latinoamericana en 2009 (véase Tabla 3). El objetivo principal es la teorización entorno a las contribuciones de las TIC-G's, los sistemas de información geográfica y los ciudadanos (Buzai, 2014). Sin embargo al analizar las tendencias en la publicación de trabajos académicos, se observa que este interés de parte de los científicos sociales, rápidamente ha sido ocupado por disciplinas científicas que responden a los vertiginosos avances tecnológicos.

Con el mejoramiento de las capacidades técnicas de las de las TIC-G's, como la posibilidad de adquisición e integración en las actividades cotidianas de los usuarios, hay un aumento de la participación ciudadana en la generación de cartografía en temas que son de su interés y preocupación. Trabajos como el de Davis y De Alencar (2011) se orientan a la validación de información obtenida a través del uso de la IGV, siendo pioneros en Latinoamérica en la evaluación de los datos. Su estudio muestra que el método de recopilación de información basado en la IGV, puede auxiliar en la producción de datos más fiables. Los estudios orientados al desarrollo de las TIC-G (Orellana y Ballari, 2009; Mateos, 2013) centran su interés en la creación de modelos eficientes de sistematización de las plataformas de gestión de datos, el uso de tecnologías, y los sistemas computacionales en temáticas como: la geovisualización y su uso en la Internet, así como la participación ciudadana. Sin embargo difieren la teorización en torno a los impactos que estos instrumentos generan en los grupos sociales.

Particularmente en 2013 se observa en el ámbito académico una tendencia a cubrir diferentes aspectos de la IGV, con la continua predominancia de las disciplinas de sistemas computaciones. Lo cual implica ventajas desde los aspectos técnicos y de gestión de datos. Esta es la ruta que ha tomado fuerza en los estudios y trabajos desarrollados sobre la IGV en Latinoamérica. En este mismo año, se observa la influencia de corrientes analíticas como la cartografía participativa, la cual incorpora a las TIC-G's o bien se basa en mapas conceptuales para el mapeo colaborativo, con trabajos como: detección de humedales; mapeo colaborativo de código abierto para la generación de cartografía urbana por medio de visualización; sistemas de recuperación de datos de las redes sociales para la generación de cartografía, así como para la validación de información remota a través de la IGV (Filho et al., 2013; De Oliveira et al., 2014; Atzmanstorfer et al., 2014; Eckle y De Albuquerque, 2015; Bravo et al., 2015).

Específicamente en el área de la infraestructura de datos espaciales, como en la validación y calidad de datos, donde existe trabajo continuo de los académicos latinoamericanos desde 2011 a la fecha. Sobresaliendo las disciplinas de los sistemas computacionales con orientación al uso de los Sistemas de Información Geográfica. En general los estudios se enfocan en la generación de cartografía funcional 
para uso oficial. El desarrollo logrado por las ciencias de la computación ha influido positivamente mejorando las herramientas de la IGV, con el desarrollo de paquetes informáticos, sistemas computacionales y servicios de geovisualización - Infraestructuras de Datos Espaciales (IDE) - , facilitando el uso amigable de estas plataformas para los usuarios (Ballari et al., 2012, 2014; Pacheco, 2015).

Desde las ciencias sociales, trabajos como los de McCall (2011) con una perspectiva de Cartografia Participativa, sitúa a la IGV como un sistema adicional generador de contenido espacial por usuarios. El autor plantea que la IGV debe construirse valorando el conocimiento local y abogando por una mayor inclusión de los diferentes grupos de la sociedad en la generación de información geográfica. Su propuesta aboga por la integración de prácticas del buen gobierno y la participación ciudadana usando como marco la IGV.

De acuerdo con la literatura revisada, es hasta 2015 cuando hay un nuevo intento para analizar los fundamentos teóricos, metodológicos y conceptuales de la IGV (McCall, 2015). Así pues, la propuesta de McCall (2011; 2015) hace una diferenciación entre el proceso de generación de información basada en los ciudadanos con un enfoque local, y el uso meramente técnico por expertos de información georreferenciada para la toma de decisiones. El autor sostiene que la participación de los usuarios se debe considerar desde la producción, validación, difusión y utilización de la información, partiendo de los preceptos y la experiencia de los sistemas de información geográfica participativa (SIGP). Desde la perspectiva crítica los Sistemas de Información Geográfica (SIG-Crítico), Güiza y McCall (2016) hacen un análisis del acceso a la tecnología en asentamientos urbanos, irregulares y pobres planteando el uso de tecnologías y estrategias de cartografía participativa usando la IGV como referencia y planteado respuestas a riesgos y emergencias.

Resultan relevantes las aplicaciones a problemáticas sociales en el campo de la IGV. Particularmente porque trata con problemáticas ambientales y sociales relevantes, en este sentido la revisión de la literatura arroja datos interesantes. El mayor número de estudios encontrados en los que se aplica IGV, se vinculan con temáticas de desastres y riesgos, especialmente inundaciones. Un amplio segmento de investigadores, particularmente del área de los sistemas computacionales desarrollan trabajos de gestión de información eficiente en caso de desastres desde una perspectiva técnica de gestión de desastres.

Concretamente Horita et al. $(2013 ; 2014)$ investigan la integración de la IGV en prácticas de logística humanitaria, para zonas afectadas por desastres. Los trabajos de Manfre et al. (2012) para la gestión de desastres y riesgos a través de los SIG, y el uso de datos espaciales obtenidos desde la IGV, tienen el objetivo de potenciar la gestión y toma de decisiones. Por otra parte, diversos estudios sobre el tema de los desastres, se encuentran relacionados con la arquitectura computacional para la toma de decisiones en momentos de crisis, combinando información convencional y 
la IGV (Horita y De Albuquerque 2013). Siguiendo la misma temática, Herfort et al. (2014) y De Albuquerque et al. (2015) desarrollan su investigación basándose en el uso de datos obtenidos desde redes sociales para el análisis de inundaciones; y en su caso Degrossi et al. (2014) y Schelhorn et al. (2014) diseñan propuestas de colaboración abierta para la gestión de riesgos por medio de la IGV.

Las contribuciones de diferentes estudiosos de los temas de la IGV en Latinoamérica en los últimos tres años va en aumento, lo que enriquecerá el panorama de estudios de este tipo. Se infiere que todavía resulta escasa la producción académica en América Latina que se distribuya en la web, lo cual implica que hayamos dejado trabajos y contribuciones en el área que son relevantes. Sin embrago, a partir de los trabajos que pudimos analizar presumimos que hay avances en aspectos tecnológicos y desarrollo de sistemas computaciones ventajosos para la IGV y concebidos para responder al contexto latinoamericano. Desde nuestra perspectiva, la predominancia de las ciencias de la computación y sistemas en temas de la IGV está relacionado con la rapidez con la que estos instrumentos cambian y mejoran sus capacidades de comunicación y desempeño. Esto impone retos a las ciencias sociales, interesadas en estudiar aspectos relativos a la trasparencia, el acceso a las tecnologías y los servicios que permiten su uso en contextos como el latinoamericano.

\section{Conclusiones}

Comprender la importancia de la participación de los usuarios en la divulgación de información cartográfica es crucial para la generación de nuevas investigaciones. La IGV es una opción para la generación y democratización de la información, y este desarrollo se relaciona con las posibilidades técnicas que ofrecen los dispositivos actuales. Es importante señalar que para el caso latinoamericano la brecha digital tiene implicaciones en los usuarios, así como las capacidades tecnológicas y de infraestructura para envío y recepción de información, además de los aspectos sociales y culturales relacionados. Todo lo cual se debe de tomar en cuenta al utilizar técnicas como la IGV.

Si bien el uso y aplicación de la IGV es emergente en América Latina, hay un creciente interés de parte de los académicos de la región que llevan a cabo investigación en la IGV, sin embargo, se observan algunas contribuciones relevantes en áreas teóricas, metodológicas, mostrando el potencial existente para las áreas científicas sociales. En la región, el modelo predominante es el anglosajón por lo cual habrá que probarse y adecuarse a las condiciones particulares del contexto latinoamericano.

Resulta claro que el futuro en la producción, validación, difusión y utilización de la información basada en la IGV, deberá de tomar en cuenta un enfoque multidisciplinario. Se observa interés de la academia latinoamericana, en el análisis del marco analítico y epistemológico de la IGV, en las prácticas sociales y en los temas 
del acceso, la trasparencia y la apropiación social de estos instrumentos, sin embargo es todavía muy poca la contribución en este tema. En América Latina, la literatura reporta investigación desarrollada principalmente en áreas de sistemas computacionales, en los cuales temas como la calidad y validación de datos están en auge en los últimos años. Asimismo continua la tendencia en la aplicación de la IGV para la gestión de riesgos y desastres, particularmente en temas como la logística para la respuesta a emergencias; asuntos que por las condiciones de vulnerabilidad social existente en América Latina seguirán acentuándose, por lo que se requiere de la participación de diferentes actores sociales y disciplinas del conocimiento.

\section{Retos futuros}

Los desafíos futuros de la IGV se encuentran en diferentes áreas. Desde la perspectiva de la neogeografía, la mayor riqueza de la IGV se encuentra en la valoración del punto de vista del usuario. El aspecto más relevante de este marco analítico, es la participación voluntaria del usuario en la generación, provisión y distribución de información geográfica. Por lo que es necesario enfatizar que hacen falta regulaciones legales que obliguen al solicitante de información que provee un servicio en la web o en las aplicaciones móviles, que de aviso a los usuarios del tipo de información que se está recogiendo y que se solicite su autorización. Lo cual desde una perspectiva analítica conceptual, implica que se tendrá que profundizar en el análisis del carácter voluntario de la provisión de información de parte del usuario, lo que conlleva a discusiones de carácter ético sobre la protección de la privacidad e identidad.

Finalmente, si bien existen esfuerzos en mejorar la validación de los datos, aún se tienen que generar sistemas óptimos que incluyan marcos conceptuales multidisciplinarios, que asocien cuestiones sociales y técnicas para la validación de la información. Temas como seguridad de datos, validación, calidad y ética en el uso de la información están presentes en el marco disciplinar emergente de la IGV. Como se mencionó antes, consolidar y estandarizar los términos y los componentes de la IGV será parte importante en futuros estudios del complejo teórico de la neogeografía.

\section{Bibliografía}

Atzmanstorfer, K. et al. (2014). "The Geocitizen-approach: community-based spatial planning - an Ecuadorian case study", Cartography and Geographic Information Science, 41(3):248-259.

Ballari, D.; Pacheco, D. y Delgado, O. (2012). "Retos para la investigación en infraestructuras de datos espaciales", Memorias del II Congreso Binacional 
de Investigación, Ciencia y Tecnología de las Universidades del Sur del Ecuador y Norte del Perú, Cuenca, pp. 594-597.

Ballari, D. et al. (2014). Tendencias en infraestructuras de datos espaciales en el contexto latinoamericano, pp. 177-184.

Bravo, J.V.M. et al. (2015). "The compatibility of metadata available at VGI systems with the metadata profile employed at the Brazilian National spatial data infrastructure (INDE-BR)", Boletim de Ciencias Geodésicas, 21(3):465-483.

Bravo, J.V.M. y Sluter, C.R. (2015). "O problema da qualidade de dados espaciais na era das informações geográficas voluntárias", Boletim de Ciências Geodésicas, 21(1):56.

Buzai, G.D. (2014). "Neogeografía y sociedad de la información geográfica una nueva etapa en la historia de la geografía", Boletín del Colegio de Geógrafos del Perú, 1:1-12.

Cardoso, S.D. et al. (2016). "SWI: A Semantic Web Interactive Gazetteer to support Linked Open Data”, Future Generation Computer Systems, 54:389-398.

Cook, I. (2005). "Positionality/situated knowledge", en Cultural geography - a critical Ddctionary of key concepts, London, pp. 16-26.

Davis, C.A. y De Alencar, R.O., (2011). "Evaluation of the quality of an online geocoding resource in the context of a large Brazilian city", Transactions in GIS, 15(6):851-868.

Davis, C.A., De Souza Vellozo, H. y Pinheiro, M.B. (2013). "A framework for web and mobile volunteered geographic information applications", Proceedings of the brazilian symposium on geoInformatics, Campos do Jordao, Brasil, National Institute for Space Research — INPE, pp. 147-157.

De Albuquerque, J.P. et al., (2015). "A geographic approach for combining social media and authoritative data towards identifying useful information for disaster management", International journal of geographical information science, 29(4):667-689.

De Brito, R.M., Degrossi, L.C. y De Albuquerque, J.P., (2015). "An experimental evaluation of a crowdsourcing-based approach for flood risk management", CIBSE 2015 - XVIII Ibero-American Conference on Software Engineering, Ibero-American Conference on Software Engineering, pp. 393-403.

De Oliveira, M.G. et al. (2014), "Automated production of volunteered geographic information from social media", Proceedings of the Brazilian Symposium on GeoInformatics, National Institute for Space Research - INPE, pp. 118-129. 
De Oliveira, M.G. et al. (2015). "Leveraging VGI for gazetteer enrichment: A case study for geoparsing twitter messages", en J. Gensel y M. Tomko (eds.), Web and wireless geographical information systems, Grenoble, France, pp. 20-36.

De Souza, W.D. et al. (2014). "ClickOnMap: a framework to develop volunteered geographic information systems with dynamic metadata", en B. Murgante et al. (eds.), Lecture Notes in Computer Science (including subseries Lecture Notes in Artificial Intelligence and Lecture Notes in Bioinformatics), Lecture Notes in Computer Science, Cham: Springer International Publishing, pp. 532-546.

Degrossi, L.C. et al. (2014). "Flood citizen observatory: a crowdsourcing-based approach for flood risk management in Brazil", International Conference on Software Engineering and Knowledge Engineering, SEKE. Knowledge Systems Institute Graduate School.

Eckle, M. and De Albuquerque, J.P. (2015). "Quality assessment of remote mapping in OpenStreetMap for disaster management purposes", ISCRAM 2015 Conference Proceedings - 12th International Conference on Information Systems for Crisis Response and Management, Information Systems for Crisis Response and Management, ISCRAM.

Filho, H.F. et al. (2013). "Teaching VGI as a strategy to promote the production of urban digital cartographic databases", Joint Urban Remote Sensing Event 2013, JURSE 2013, pp. 222-225.

Gonzalez, A.L. et al. (2013). "OurMap: Representing crowdsourced annotations on geospatial coordinates as Linked Open Data", Lecture Notes in Computer Science (including subseries Lecture Notes in Artificial Intelligence and Lecture Notes in Bioinformatics), 8224 LNCS(i), pp.77-93.

Goodchild, M.F. (2007a), "Citizens as sensors: the world of volunteered geography”, GeoJournal, 69(4):211-221.

(2007b). "Citizens as sensors: web 2.0 and the volunteering of geographic information", Geofocus, 7:8-10.

Güiza, F. and McCall, K.M. (2016). "El enfoque crítico del análisis geoespacial. Deconstruyendo la vulnerabilidad en una comunidad afectada por inundaciones en la ciudad de Morelia”, en J.F. Mas-Causel and J.C. Ley (eds.), El análisis geoespacial en los estudios urbanos (en prensa).

Herfort, B. et al., (2014). "Exploring the geographical relations between social media and flood phenomena to improve situational awareness: A study about the river Elbe flood in June 2013”, en J. Huerta, S. Schade y C. Granell (eds.) Lecture Notes in Geoinformation and Cartography, Lecture Notes in Geoin- 
formation and Cartography. Cham, Springer International Publishing, pp. 55-71.

Herfort, B. et al. (2015). "Towards assessing the quality of Volunteered Geographic Information from OpenStreetMap for identifying critical infrastructures", ISCRAM 2015 Conference Proceedings - 12th International Conference on Information Systems for Crisis Response and Management. Information Systems for Crisis Response and Management, ISCRAM.

Hirata, E. et al., (2013). "Mapeamento dinâmico e colaborativo de alagamentos na cidade de Sao Paulo", Boletim de Ciências Geodésicas, 19(4):602-623.

Horita, F.E.A. et al., (2013). "The use of volunteered geographic information and crowdsourcing in disaster management: A systematic literature review", 19th Americas Conference on Information Systems, AMCIS 2013 - Hyperconnected World: Anything, Anywhere, Anytime, pp. 3539-3548.

Horita, F.E.A. and De Albuquerque, J.P. (2013). "An approach to support decisionmaking in disaster management based on volunteer geographic information (VGI) and spatial decision support systems (SDSS)", ISCRAM 2013 Conference Proceedings - 10th International Conference on Information Systems for Crisis Response and Management. Karlsruher Institut fur Technologie (KIT), pp. 301-306.

Horita, F.E.A. et al. (2014). "A framework for the integration of volunteered geographic information into humanitarian logistics", 20th Americas conference on information systems, AMCIS 2014, Savannah, Georgia, USA, Association for Information Systems, p. 12.

Horita, F.E.A. et al. (2015). "Development of a spatial decision support system for flood risk management in Brazil that combines volunteered geographic information with wireless sensor networks", Computers \& Geosciences, 80:8494.

Manfre, L.A. et al. (2012). "An Analysis of Geospatial Technologies for Risk and Natural Disaster Management", ISPRS International Journal of GeoInformation, 1(3):166-185.

Mateos, P., (2013). “Geovisualización de la población: nuevas tendencias en la web social”, Investigaciones Geográficas, 60:87-100.

McCall, M.K. (2011). "Participacion y buena gobernanza en cartografia social y SIGP, las contribuciones de la neogeografia y la IGV (VGI)", Seminario Geografía Crítica: territorialidad, espacio y poder en América Latina, Bogotá, pp. 128-149.

McCall, M.K.; Martinez, J. and Verplanke, J. (2015). "Shifting boundaries of volunteered geographic information systems and modalities: Learning from 
PGIS", ACME: An International E-Journal for Critical Geographie, 14(3):791-826.

Miranda, T.S. et al. (2012). "Volunteered geographic information in the context of local Spatial Data Infrastructures", Urban and Regional Data Management, UDMS Annual 2011 - Proceedings of the Urban Data Management Society Symposium 2011. pp. 123-138.

Orellana, D. and Ballari, D. (2009). "La GeoWeb y su evolución: un marco de análisis en tres dimensiones", Revista de la Universidad del Azuay, 49:25-52.

Pacheco, D. \& Chester, P., (2012). "Desarrollo, implementación y publicación de un sistema geográfico para la gestión vial del cantón Cuenca", Memorias del II Congreso Binacional de Investigación, Ciencia y Tecnología de las Universidades del Sur del Ecuador y Norte del Perú, Cuenca, pp. 598-606.

Pacheco, D., (2013). Infraestructuras de datos espaciales en dispositivos móviles inteligentes, Universidad de Azuay.

Pacheco, D., (2015). "Evolución de la infraestructura de datos espaciales en la Universidad del Azuay", Revista de la Universidad del Azuay, Ecuador, pp. 237256.

Peters, R. and De Albuquerque, J.P. (2015). "Investigating images as indicators for relevant social media messages in disaster management", ISCRAM 2015 Conference Proceedings - 12th International Conference on Information Systems for Crisis Response and Management. Information Systems for Crisis Response and Management, ISCRAM.

Poorazizi, M., Hunter, A. and Steiniger, S. (2015). “A Volunteered Geographic Information Framework to Enable Bottom-Up Disaster Management Platforms", ISPRS International Journal of Geo-Information, 4(3):1389-1422.

Saber.cic.ipn.mx (2016). "SABER", online, 1, 2016. Available at: <http://www. saber.cic.ipn.mx/cake/SABERsvn/trunk/docentes/webView/25> [Accessed 13 Marz. 2016]. [Accedido marzo 13, 2016].

Schelhorn, S.J. et al. (2014). "Identifying elements at risk from OpenStreetMap: The case of flooding", Proceedings - 11th International Conference on Information Systems for Crisis Response and Management. The Pennsylvania State University, pp. 508-512.

Souza, W.D. et al. (2013). "DM4vGI: A template with dynamic metadata for documenting and validating the quality of volunteered geographic information", Proceedings of the Brazilian Symposium on GeoInformatics. National Institute for Space Research - INPE, pp. 1-12. 
Stasch, C. et al. (2009). A stimulus-centric algebraic approach to sensors and observations, N. Trigoni, A. Markham and S. Nawaz (eds.), Berlin, Heidelberg, Springer Berlin Heidelberg.

Vidal-Filho, J.N. et al. (2013). "Qualitative analysis of volunteered geographic information in a spatially enabled society project", en B. Murgante et al. (eds.), Lecture Notes in Computer Science (including subseries Lecture Notes in Artificial Intelligence and Lecture Notes in Bioinformatics), Lecture Notes in Computer Science. Berlin, Heidelberg, Springer Berlin Heidelberg, pp. 378-393. 